\title{
Review of: "Bagaza virus in wild birds, Portugal, $2021 "$
}

\author{
Muthannan Andavar Ramakrishnan
}

Potential competing interests: The author(s) declared that no potential competing interests exist.

\section{Abstarct}

- in one corn bunting and several red-legged partridges .. remove one and several

\section{Introduction}

- one corn bunting (Emberiza calandra) change to a corn bunting

\section{The study}

- On September 15t, 2021, change to September 1, 2021

- ten wild birds (nine partridges and one corn bunting) change to ten wild birds (9 partridges and 1 corn bunting)

- IREC and CIBIO-InBIO.

- Table 2: change into Table 1. This table is blurred and not readable. Provide the new high resolution Table.

- he sighting reports of partridges with compatible neurological signs such as disorientation and motor incoordination were also reported locally (specimens not collected). If specimens not collected then how laboratory examinations carried out?

- Official diagnosis was carried later, --- this may need to changed as further confirmation was carried out.

- Overall, the study section needs to be rewritten For example; in the present study two outbreaks were investigated. The first outbreak occurred during September 1, 2021... three red-legged partridges were found dead in a hunting ground in Serpa, southern Portugal. The second outbreaks occurred....

- In addition, growing feathers were collected from 30 partridges live-trapped in the same area on October $3^{\text {rd }}$, using cages with wheat bait (Permission number 4 - DGVF/DRCA/ 2021). In which area? Then sample number need to be changed. For example, samples were collected from 12 dead birds and 30 live birds etc.

- using the a method previously described --- grammatical corrections will improve the quality of the MS.

- The amplicons were purified ... gel or PCR purified?

- The results showed that eight red-legged partridges and one corn bunting out of the 12 wild birds found dead (66.7\%), and four out of 30 live-captured red-legged partridges (13.3\%) tested positive for BAGV

.... Write numerical such as 8, 1

- confirming the cause of death ??? avoid this sentence

- $100 \%$ similarity ... use identity 
- MEGA software - write the version also

- These were based on concatenated.. remove concatenated

- Upon necropsy, all birds were in good body condition suggesting an acute course of disease. Histopathology albeit hampered by autolysis and freezing artefacts revealed lymphoid... this is contrasting statement... good body condition vs autolysis

\section{Discussion and Conclusions}

- (REVIVE) - please expand

- Final question? Why whole genome sequencing not tried.

In brief, a good study but needs thorough revision. All the best authors. 